\title{
MUON COLLIDERS: THE ULTIMATE NEUTRINO BEAMLINES
}

\author{
Bruce J. King, Brookhaven National Laboratory ${ }^{1}$
}

\begin{abstract}
It is shown that muon decays in straight sections of muon collider rings will naturally produce highly collimated neutrino beams that can be several orders of magnitude stronger than the beams at existing accelerators. We discuss possible experimental setups and give a very brief overview of the physics potential from such beamlines. Formulae are given for the neutrino event rates at both short and long baseline neutrino experiments in these beams.
\end{abstract}

\section{INTRODUCTION}

Recent feasibility studies and design work for muon colliders [1] has begun to focus attention on the exciting physics possibilities from the uniquely intense neutrino beams at proposed muon storage rings (muSR) that will use muon collider technology to produce and store large muon currents. Such muSR include both storage rings dedicated to neutrino physics and the parasitic use of the accelerator and collider rings of muon colliders themselves.

\section{POTENTIAL FOR NEUTRINO PHYSICS}

The neutrino beamlines can be used for two sorts of neutrino experiments:

1. short baseline (SB) experiments, where the detector is placed close to the neutrino source to obtain the most intense beam possible and hence gather very high event statistics of neutrino interactions.

2. long baseline (LB) experiments, where a very massive neutrino detector is placed far away from the neutrino source, deliberately sacrificing event rate in order to study baseline-dependent properties of the neutrinos and, in particular, whether there are "flavor oscillations" in the types of neutrinos composing the beam.

The large muon currents and tight collimation of the neutrinos results in extremely intense beams - intense enough even to constitute a potential off-site radiation hazard [2]. This gives several advantages over the neutrino beams produced today from pion decay at accelerator beamlines:

1. event statistics for short-baseline (SB) experiments that might be three or more orders-of-magnitude larger than in today's high-rate neutrino experiments

\footnotetext{
1 web page: http://pubweb.bnl.gov/people/bking email: bking@bnl.gov. This work was performed under the auspices of the U.S. Department of Energy under contract no. DE-AC02-98CH10886.
}

2. both higher statistics and longer baselines for long baseline (LB) experiments

3. extremely well understood and pure two-component beams with accurately predictable energy spectra, angular divergences and intensities

4. the first high flux electron-neutrino and electronantineutrino beams at high energies

5. the possibility of tuning the flavor composition of the beam by varying the polarization direction of the muons.

Recent evidence of neutrino oscillations from several different experiments has resulted in much recent interest in LB neutrino oscillation experiments. LB experiments at muSR have the potential $[3,4,5]$ to either convincingly refute or precisely characterize much of today's evidence for neutrino oscillations.

SB experiment have a different physics motivation, involving several precision measurements that can contribute mainly to our understanding of the interactions between elementary particles. In this respect, they have the potential $[3,4,5]$ to make important measurements have comparable value to (and are complementary to) some of the best precision measurements at today's colliders: LEP, SLC, CLEO, Tevatron, HERA and the B factories that are now coming on-line.

The huge increase in beam intensity allows the use of novel high-performance SB neutrino detectors that are capable of much better reconstructing neutrino events than today's huge high-rate neutrino detector targets. An example of such a detector [3] is shown in figure 1 .

\section{NEUTRINO PRODUCTION AND EVENT RATES}

Neutrinos are emitted from the decay of muons in the collider ring:

$$
\begin{aligned}
& \mu^{-} \rightarrow \nu_{\mu}+\overline{\nu_{\mathrm{e}}}+\mathrm{e}^{-}, \\
& \mu^{+} \rightarrow \overline{\nu_{\mu}}+\nu_{\mathrm{e}}+\mathrm{e}^{+} .
\end{aligned}
$$

The thin pencil beams of neutrinos for experiments will be produced from long straight sections in either the collider ring or a ring dedicated to neutrino physics. From relativistic kinematics, the forward hemisphere in the muon rest frame will be boosted, in the lab frame, into a narrow cone with a characteristic opening half-angle, $\theta_{\nu}$, given in obvious notation by

$$
\theta_{\nu} \simeq \sin \theta_{\nu}=1 / \gamma=\frac{m_{\mu}}{E_{\mu}} \simeq \frac{10^{-4}}{E_{\mu}(\mathrm{TeV})} .
$$




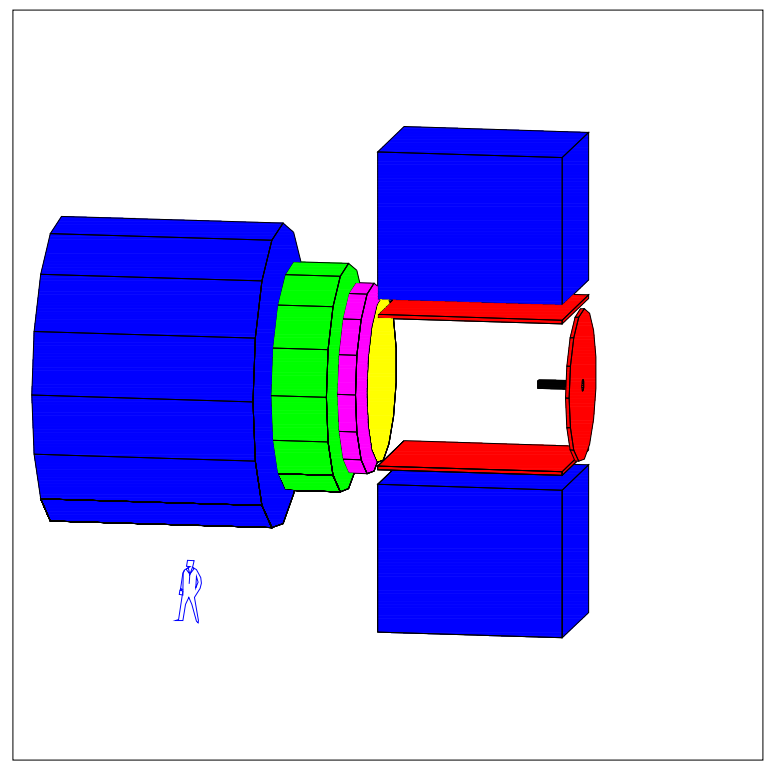

Figure 1: Example of a general purpose SB neutrino detector. A human figure in the lower left corner illustrates its size. The neutrino target is the small horizontal cylinder at mid-height on the right hand side of the detector. Its radial extent corresponds roughly to the radial spread of the neutrino pencil beam, which is incident from the right hand side. Further details are given in reference [3].

The decays of muons into neutrinos are very well understood and it is possible [6] to derive simple, approximate quantitative expressions for the neutrino fluences at experiments and the corresponding experimental event rates in a given target.

The neutrino targets at SB experiments might reasonably cover the boosted forward hemisphere of the neutrino beam. Besides the beam properties, the event rate depends on the depth of the neutrino target, $l$, in units of $\mathrm{gm} . \mathrm{cm}^{-2}$ and on the number of years of data-taking, Y. Thus, a convenient benchmark for the size of neutrino event samples the beam can produce is $\mathrm{N}^{\mathrm{sb}}$, defined as

$$
\begin{array}{r}
\text { no. of } \nu \text { events }=\mathrm{N}^{\mathrm{sb}}\left[\text { events } / \mathrm{yr} /\left(\mathrm{gm}_{\mathrm{cm}} \mathrm{cm}^{-2}\right]\right) \times \\
l\left[\mathrm{gm} . \mathrm{cm}^{-2}\right] \times \mathrm{Y},
\end{array}
$$

Ref [6] derives the following numerical expression for $\mathrm{N}^{\text {sb }}$ in SB detectors satisfying a couple of reasonable assumptions:

$$
\begin{aligned}
& \mathrm{N}^{\text {sb }}\left[\text { events } / \mathrm{yr} /\left(\mathrm{gm}_{\mathrm{cm}} \mathrm{cm}^{-2}\right]\right) \simeq 2 \times 10^{-15} \times \\
& E_{\mu}[\mathrm{GeV}] \times N_{\mu}^{s s}\left[y r^{-1}\right],
\end{aligned}
$$

where the parameter $N_{\mu}^{s s}$ is the number of forward-going muons decaying in the production straight section per year.

Similarly, the number of LB events depends on the neutrino beam, the baseline distance, $\mathrm{L}$, from neutrino source to detector and the detector mass $(\mathrm{L})$ through:

$$
\begin{array}{r}
\text { no. of } \nu \text { events }= \\
\mathrm{N}^{\mathrm{lb}}\left[\text { events } / \mathrm{yr} /\left(\mathrm{kg} \cdot \mathrm{km}^{-2}\right)\right] \times \frac{\mathrm{M}[\mathrm{kg}]}{(\mathrm{L}[\mathrm{km}])^{2}} \times \mathrm{Y},
\end{array}
$$

with

$$
\begin{array}{r}
\mathrm{N}^{\mathrm{lb}}\left[\text { events } / \mathrm{yr} /\left(\mathrm{kg} \cdot \mathrm{km}^{-2}\right)\right] \simeq \\
1.6 \times 10^{-20} \times N_{\mu}^{s s}\left[y r^{-1}\right] \times \times\left(E_{\mu}[\mathrm{GeV}]\right)^{3}
\end{array}
$$

(The parameter, $\gamma \cdot \delta \theta$ has value 1 except for neutrino beams from muon beams with large angular divergences.)

Reference [6] also tabulates the expected event rates for plausible muon collider scenarios. These predictions are reproduced as table 1. Extraordinary event samples of $10^{10}$ interactions appear plausible for SB experiments at muSR with muon energies of order $100 \mathrm{GeV}$ or above.

\section{CONCLUSIONS}

In conclusion, the essentially free neutrino beamlines at muon colliders and the beams from other muSR using muon collider technology could provide happy prospects for the future of neutrino physics.

\section{REFERENCES}

[1] The Muon Collider Collaboration, "Status of Muon Collider Research and Development and Future Plans", to be submitted to Phys. Rev. E.

[2] B.J. King, "Potential Hazards from Neutrino Radiation at Muon Colliders", these proceedings.

[3] B.J. King, "Neutrino Physics at a Muon Collider", Proc. Workshop on Physics at the First Muon Collider and Front End of a Muon Collider, Fermilab, November 6-9, 1997. Available at http://pubweb.bnl.gov/people/bking/.

[4] B.J. King, "Neutrino Physics at Muon Colliders", Proc. 4th Int. Conf. on Workshop on the Physics Potential and Development of Muon Colliders, San Francisco, December 1997, ed. David B. Cline. Available at http://pubweb.bnl.gov/people/bking/.

[5] B.J. King, "Muon Colliders: New Prospects for Precision Physics and the High Energy Frontier", BNL CAP224-MUON-98C, Proc. Latin Am. Symp. on HEP, April 8-11,1998, San Juan, Puerto Rico, Ed. J.F. Nieves. Available at http://pubweb.bnl.gov/people/bking/.

[6] I. Bigi et al. "An Overview of the Potential for Neutrino Physics at Future Muon Collider Complexes". Work in progress. Contact B.J. King, email: bking@bnl.gov . 
Table 1: Predicted neutrino fluxes and event rates for muSR's or muon colliders at several energies.

\begin{tabular}{|r|cccccc|}
\hline muon energy, $\mathrm{E}_{\mu}$ & $2 \mathrm{GeV}$ & $20 \mathrm{GeV}$ & $50 \mathrm{GeV}$ & $175 \mathrm{GeV}$ & $500 \mathrm{GeV}$ & $5 \mathrm{TeV}$ \\
\hline$\left(\mu^{-}\right.$or) $\mu^{+} /$year, $\mathrm{N}_{\mu}\left[10^{20}\right]$ & 3.0 & 3.0 & 6.0 & 6.0 & 3.2 & 3.6 \\
fract. str. sect. length, $\mathrm{f}_{\mathrm{ss}}$ & 0.40 & 0.30 & 0.12 & 0.12 & 0.12 & 0.03 \\
$\nu$ ang. diverg., $\gamma \cdot \delta \theta_{\nu}$ & 1 & 1 & 1 & 1 & 10 & 1 \\
time to beam dump, $\mathrm{t}_{\mathrm{D}}\left[\gamma \tau_{\mu}\right]$ & no dump & no dump & no dump & no dump & 0.5 & no dump \\
$N^{s b}\left[\mathrm{evt} / \mathrm{yr} /\left(\mathrm{g} . \mathrm{cm}^{-2}\right)\right]$ & $5.1 \times 10^{5}$ & $3.8 \times 10^{6}$ & $6.5 \times 10^{6}$ & $2.7 \times 10^{7}$ & $2.3 \times 10^{7}$ & $1.0 \times 10^{8}$ \\
$l \times Y$ [gm $\left./ \mathrm{cm}^{2}\right]$ for $10^{10}$ events & $2.0 \times 10^{4}$ & 2600 & 1500 & 370 & 430 & 100 \\
$N^{l b}\left[\mathrm{evt} / \mathrm{yr} /\left(\mathrm{kg} . \mathrm{km}^{-2}\right]\right.$ & 16 & $1.2 \times 10^{4}$ & $1.4 \times 10^{5}$ & $6.2 \times 10^{6}$ & $5.0 \times 10^{5}$ & $2.2 \times 10^{10}$ \\
\hline
\end{tabular}

